\title{
Integer QP Relaxation Based Algorithms for ICI Reduction in OFDM Systems
}

\author{
Y. H. Zhang, W.-S. Lu, and T. A. Gulliver \\ Department of Electrical and Computer Engineering, University of Victoria \\ P.O. Box 3055, Victoria, B.C., Canada V8W 3P6
}

\begin{abstract}
In a fast fading channel, Doppler spread caused by user mobility destroys the orthogonality among orthogonal frequency division multiplexing (OFDM) subcarriers, resulting in intercarrier interference (ICI). In this paper, the OFDM ICI reduction problem is first formulated as a combinatorial optimization problem with integer constraints. Two relaxation methods are then utilized to relax the maximum likelihood (ML) detection problem into convex quadratic programming (QP) problems. To further reduce computational complexity, the QP problems are solved by limiting the search to a 2-dimensional subspace. A low-bit descent search (LBDS) can also be employed to improve the system performance. Performance results are given which demonstrate that the integer $Q P$ relaxation based algorithms provide excellent performance with reasonable computational complexity.
\end{abstract}

\section{INTRODUCTION}

Orthogonal frequency division multiplexing (OFDM) modulation has been widely used in communication systems to meet the demand for ever increasing data rates with low receiver complexity. It has the ability to provide high data rate transmission over frequency selective channels. Several standards have adopted OFDM as the modulation scheme, including digital audio broadcasting (DAB), digital video broadcasting (DVB), IEEE 802.11 and 802.16 [1].

In an OFDM system, the data stream is divided into $N$ parallel lower-rate data streams and multiplexed onto a number of subcarriers using an inverse fast Fourier transform (IFFT). These subcarriers are overlapped orthogonally to provide bandwidth efficient transmission. However, in a rapidly fading environment, the channel can be time-varying, even within one symbol duration. Doppler spread caused by user mobility then destroys the orthogonality among subcarriers, resulting in intercarrier interference (ICI) and degraded system performance [2] - [5].

Various algorithms have been proposed to mitigate the ICI and improve system performance over doubly-selective channels [2]-[4]. In [2], Li and Cimini provide universal bounds on the ICI in an OFDM system over time-varying fading channels, which can be evaluated and compared with the exact ICI. In [3], a decision feedback (DF) equalizer algorithm utilizing signals from several nearby subcarriers to eliminate the ICI for a certain subcarrier is described. Furthermore, an ICI suppression algorithm using parallel canceling with frequencydomain equalization techniques is presented in [4].

Based on the maximum likelihood (ML) criterion, the OFDM ICI reduction problem can be formulated as a combinatorial optimization problem with integer constraints. It has been shown that in the multiuser detection of DS-CDMA systems [7] such a problem can be solved more efficiently by using suboptimal detectors. In this paper, two relaxation methods are utilized to convert the ICI reduction problem into convex quadratic programming $(\mathrm{QP})$ problems. To further reduce the computational complexity, the QP problems can be solved by limiting the search to the 2-dimensional subspace spanned by its steepest-descent and Newton directions. A lowbit descent search (LBDS) can also be employed to improve the system performance. Performance results demonstrate that the integer QP relaxation based algorithms provide excellent performance with reasonable computational complexity.

\section{SYSTEM MODEL}

In an OFDM system, the bandwidth is split into $N$ orthogonal subchannels. The lower-rate parallel data streams employ quadrature amplitude modulation (QAM) or phase-shift keying (PSK), and the transmitted signals are modulated onto the $N$ subcarriers via an IFFT

$$
x_{n}=\frac{1}{\sqrt{N}} \sum_{k=0}^{N-1} X_{k} \exp \left(\frac{j 2 \pi k n}{N}\right) \text { for } n=0, \ldots N-1
$$

where $x_{n}$ is the time-domain signal at the $n$th sampling instant, and $X_{k}$ is the frequency-domain symbol at the $k$ th subcarrier. Equation (1) can be written in vector form as

$$
\mathbf{x}=\mathbf{F X}
$$

where $\mathbf{x}=\left[\begin{array}{llll}x_{0} & x_{1} & \cdots & x_{N-1}\end{array}\right]^{T}$ and $\mathbf{X}=\left[\begin{array}{llll}X_{0} & X_{1} & \cdots & X_{N-1}\end{array}\right]^{T}$ represent the time-domain and frequency-domain OFDM symbols, respectively, and $\mathbf{F}$ is the IFFT matrix with elements $f_{n, k}=\frac{1}{\sqrt{N}} \exp \left(\frac{j 2 \pi k n}{N}\right)$. The OFDM symbol duration is denoted by $T_{s}$, so the chip duration of each subchannel is $T_{c}=T_{s} / N$.

In this paper, we adopt a doubly frequency selective fading channel model. Thus, we have a wide sense stationary uncorrelated scattering (WSSUS) channel with impulse response given by

$$
h(t ; \tau)=\sum_{d=1}^{D} h\left(t ; \tau_{d}\right) \delta\left(\tau-\tau_{d}\right)
$$

where $\tau_{d}$ is the $d$ th path delay with $\tau_{1}<\tau_{2}<\ldots<\tau_{D}$. In a rich scattering environment, the channel autocorrelation function is separable in terms of time and delay [3]. 
A discrete version of the WSSUS channel in (3) can be modeled as a tapped delay line (TDL) with random taps [9]

$$
h(n ; l)=\sum_{d=1}^{D} h\left(n T_{c} ; \tau_{d}\right) \operatorname{sinc}\left(\frac{\tau_{d}}{T_{c}}-l\right)
$$

where $h(n ; l)$ denotes the channel coefficient for the $l$ th tap at the $n$th sampling instant, $n=0, \ldots, N-1, l=0, \ldots, L-1$ with $L=\left\lfloor\tau_{D} / T_{c}\right\rfloor+1$, and the delay between two taps is $T_{c}$.

We assume that a cyclic prefix (CP) is inserted at the beginning of each OFDM symbol before transmission, and the length $N_{p}$ of the cyclic prefix is greater than or equal to that of the channel impulse response to eliminate the intersymbol interference. Thus, the discrete signal at the receiver can be expressed as

$$
y_{n}=\sum_{l=0}^{L-1} h(n, l) x(n-l)+w_{n} \text { for } n=-N_{p}, \ldots N-1
$$

where $w_{n}$ is additive white Gaussian noise (AWGN) with zero mean and variance $\sigma^{2}$. In vector form, (5) can be written as

$$
\mathbf{y}=\mathbf{H x}+\mathbf{w}
$$

where $\mathbf{y}$ and $\mathbf{w}$ denote the time-domain received signal and AWGN noise, respectively, and $\mathbf{H}$ is the channel matrix. After removing the $\mathrm{CP}$ and performing a fast Fourier transform (FFT), we obtain

$$
\mathbf{Y}=\mathbf{A X}+\mathbf{W}
$$

where $\mathbf{Y}=\left[\begin{array}{lll}Y_{0} & \cdots & Y_{N-1}\end{array}\right]^{T}$ is the frequency-domain received signal, $\mathbf{A}=\mathbf{F}^{H} \mathbf{H F}, \mathbf{W}=\mathbf{F}^{H} \mathbf{w}$, and $H$ denotes Hermitian transpose.

If $h\left(t ; \tau_{d}\right)$ in (3) remains constant within one OFDM symbol duration, then no ICI will occur. Conversely, if the channel varies within one OFDM symbol, the orthogonality of the subcarriers does not hold, and the received signal contains both the transmitted signal and ICI from other subcarriers [5].

\section{Problem Formulation and Relaxation}

Based on the ML detection criterion, the ICI reduction problem in OFDM systems can be formulated as the optimization problem

$$
\begin{aligned}
\operatorname{minimize} & \|\mathbf{Y}-\mathbf{A X}\|_{2}^{2} \\
\text { subject to: } & X_{k} \in \mathcal{M}, \text { for } k=0,1, \ldots, N-1
\end{aligned}
$$

where $\mathcal{M}$ contains the constellation points according to the modulation being used.

The variables in (8) are complex-valued. If we define $\mathbf{Y}=\mathbf{Y}_{\mathbf{r}}+\mathbf{j} \mathbf{Y}_{\mathbf{i}}, \mathbf{A}=\mathbf{A}_{\mathbf{r}}+\mathbf{j} \mathbf{A}_{\mathbf{i}}$, and $\mathbf{X}=\mathbf{X}_{\mathbf{r}}+\mathbf{j} \mathbf{X}_{\mathbf{i}}$, then (8) can be reformulated into an optimization problem with realvalued variables as

$$
\begin{aligned}
\operatorname{minimize} & \|\hat{\mathbf{Y}}-\hat{\mathbf{A}} \mathbf{z}\|_{2}^{2} \\
\text { subject to: } & z_{k} \in \hat{\mathcal{M}} \text {, for } k=0,1, \ldots, N-1
\end{aligned}
$$

where $\hat{\mathbf{Y}}=\left[\begin{array}{l}\mathbf{Y}_{\mathbf{r}} \\ \mathbf{Y}_{\mathbf{i}}\end{array}\right], \mathbf{z}=\left[\begin{array}{l}\mathbf{X}_{\mathbf{r}} \\ \mathbf{X}_{\mathbf{i}}\end{array}\right]$, and $\hat{\mathbf{A}}=\left[\begin{array}{cc}\mathbf{A}_{\mathbf{r}} & -\mathbf{A}_{\mathbf{i}} \\ \mathbf{A}_{\mathbf{i}} & \mathbf{A}_{\mathbf{r}}\end{array}\right]$.

In what follows, the OFDM system is assumed to employ 4QAM modulation, which corresponds to $\hat{\mathcal{M}}=\{ \pm 1\}$. Clearly,
(9) is a quadratic optimization problem with discrete variables and can be expressed as

$$
\begin{gathered}
\operatorname{minimize} \mathbf{z}^{T} \mathbf{Q z}+\mathbf{q}^{T} \mathbf{z} \\
\text { subject to: } z_{k}=\{-1,1\}, \text { for } k=0, \ldots, 2 N-1
\end{gathered}
$$

where $\mathbf{Q}=\hat{\mathbf{A}}^{T} \hat{\mathbf{A}}$, and $\mathbf{q}=-2 \hat{\mathbf{A}}^{T} \hat{\mathbf{Y}}$.

\section{A. Convex relaxation}

Since the vector $\mathbf{z}$ in the ML problem (10) is a discrete set, we have a combinatorial problem with exponential computational complexity that becomes prohibitive even for a moderate number of variables. It has been shown [7] that this type of ML detection problem can be solved more efficiently by expanding the discrete feasible set into a continuous and convex feasible region. In this paper, two convex relaxation methods are utilized that allow us to consider convex QP problems that admit a fast solution which yields good performance. The first QP problem minimizes a convex quadratic objective function subject to the solution being contained within an $n$-dimensional box centered at the origin. The second QP problem minimizes the same objective function subject to the solution being contained within an $n$-dimensional ball.

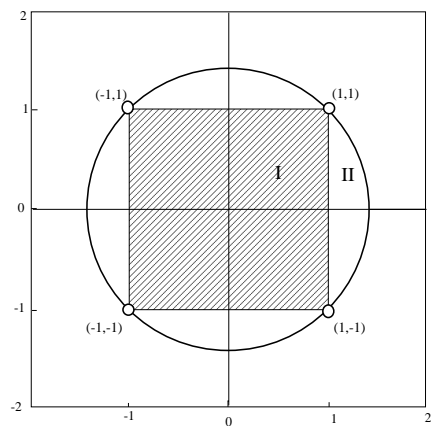

Fig. 1. The feasible set defined by (10b) (points on the circle), the feasible region defined by (11b) (I), and the feasible region defined by (12b) (I+II)

1) Bounded constraint relaxation: The discrete constraints in (10b) imply that $-1 \leq z_{k} \leq 1$, for $k=0, \ldots, 2 N-1$. Thus the ICI reduction problem (10) can be relaxed into a bounded constraint optimization problem

$$
\begin{gathered}
\operatorname{minimize} \mathbf{z}^{T} \mathbf{Q z}+\mathbf{q}^{T} \mathbf{z} \\
\text { subject to: }-1 \leq z_{k} \leq 1, \text { for } k=0, \ldots, 2 N-1
\end{gathered}
$$

The feasible region in (11b) is a $n$-dimensional hypercube centered at the origin with linear constraints. Thus the problem (11) is a convex QP problem which can be solved efficiently to provide suboptimal performance to that of (10).

2) Quadratic convex relaxation: The constraints in (10b) imply that $z^{T} z=2 N$, which is associated with the feasible region of a $2 N$-dimensional ball centered at the origin with radius $\sqrt{2 N}$. If we expand such a feasible region within the ball, the ICI reduction problem (10) is relaxed into the problem

$$
\begin{aligned}
& \operatorname{minimize} \mathbf{z}^{T} \mathbf{Q z}+\mathbf{q}^{T} \mathbf{z} \\
& \text { subject to: } \mathbf{z}^{T} \mathbf{z} \leq 2 N
\end{aligned}
$$


The problem (12) is to minimize a quadratic objective function over a convex feasible region, thus, it is a convex QP minimization problem. A unique global solution can be obtained using efficient interior-point QP solvers with reduced computational complexity.

The feasible regions of the problems (10), (11) and (12) are depicted in Fig. 1. Efficient optimization algorithms are available in the literature [8] to solve the minimization problems (11) and (12). Once the solution $\mathbf{z}^{*}$ of (11) or (12) is obtained, the solution of (10) can be approximated as $\operatorname{sign}\left(\mathbf{z}^{*}\right)$.

\section{B. 2-dimensional search method}

To further reduce computational complexity, the solutions of (11) or (12) can be obtained by limiting the search to the 2dimensional subspace spanned by its steepest-descent direction (i.e., negative gradient of the objective function) and Newton direction. In doing so, we set

$$
\mathbf{z}=\eta_{1} \mathbf{v}_{1}+\eta_{2} \mathbf{v}_{2}
$$

where $\mathbf{v}_{1}=\mathbf{q}, \quad \mathbf{v}_{2}=\mathbf{Q}^{-1} \mathbf{q}$, and $\eta_{1}, \eta_{2}$ are two scalar variables. Then the problem (11) is converted to the 2dimensional problem

$$
\begin{gathered}
\operatorname{minimize} \boldsymbol{\eta}^{T} \mathbf{S} \boldsymbol{\eta}+\mathbf{p}^{T} \boldsymbol{\eta} \\
\text { subject to : }-1 \leq V_{k} \boldsymbol{\eta} \leq 1
\end{gathered}
$$

where $\boldsymbol{\eta}=\left[\begin{array}{ll}\eta_{1} & \eta_{2}\end{array}\right]^{T}, \mathbf{S}=\mathbf{V}^{T} \mathbf{Q V}, \mathbf{p}=\mathbf{V}^{T} \mathbf{q}, V_{k}$ is the $k$ th row of the matrix $\mathbf{V}$, and $\mathbf{V}=\left[\begin{array}{ll}\mathbf{v}_{1} & \mathbf{v}_{2}\end{array}\right]$.

Similarly, the problem (12) can be reformulated to the 2dimensional problem

$$
\begin{array}{r}
\operatorname{minimize} \boldsymbol{\eta}^{T} \mathbf{S} \boldsymbol{\eta}+\mathbf{p}^{T} \boldsymbol{\eta} \\
\text { subject to }: \boldsymbol{\eta}^{T} \mathbf{R} \boldsymbol{\eta} \leq 2 N
\end{array}
$$

where $\mathbf{R}=\mathbf{V}^{T} \mathbf{V}$. If we denote the solution of the problem (14) or (15) as $\boldsymbol{\eta}^{*}$, the solution $\mathbf{z}^{*}$ of problem (11) or (12) can be calculated using (13) accordingly, and $\operatorname{sign}\left(\mathbf{z}^{*}\right)$ is then taken as the solution of (10).

\section{Performance Enhancement by Low-Bit Descent Search}

In LBDS, a given binary sequence is associated with an objective function to be minimized. The search process evaluates, compares, and determines the optimal sign switches of a relatively small number of sequence components to yield maximum reduction in the objective function in (10). LBDS has been applied recently to various problems [10]. As will be demonstrated, the performance of the proposed algorithm can be considerably enhanced using 1-bit, 2-bit, or a combined 1-bit-and-2-bit LBDS, at an insignificant increase in computational complexity.

From [10], the one-bit descent search can be carried out by evaluating $\mathbf{z} \odot \boldsymbol{\xi}$ (here $\odot$ denotes component-wise multiplication), where $\boldsymbol{\xi}=\tilde{\mathbf{Q}} \mathbf{z}+\mathbf{q} / \mathbf{2}$, and $\tilde{\mathbf{Q}}$ is generated from $\mathrm{Q}$ with its diagonal components set to zero. Index $k^{*}$ is then identified as where the corresponding component $\xi_{k^{*}}$ has maximum value, and the sign of $z_{k^{*}}$ is switched to obtain an improved solution. Similarly, a 2-bit LBDS is performed by computing $\mathbf{G}=\boldsymbol{\xi} \boldsymbol{e}^{T}+\mathbf{e}^{T} \boldsymbol{\xi}-2 \mathbf{Q} \odot\left(\mathbf{z z}^{T}\right)$, where $\mathbf{e}$ is the all-one vector. The index $\left(k^{*}, m^{*}\right)$ is identified as where the component $P_{k^{*}, m^{*}}$ reaches its maximum value, and an improved solution is then obtained by switching the signs of the $k^{*}$ th and $m^{*}$ th components of $\mathbf{z}^{*}$.

\section{PERFormance EVAluation}

The proposed algorithms were applied to an OFDM system with $N=64$ subcarriers. The length of the cyclic prefix was chosen to be $N_{p}=N / 8$. We assume 4-QAM modulation with a system bandwidth of $200 \mathrm{kHz}$. A two-tap WSSUS fading channel was employed, where each channel tap was an independent complex Gaussian random process with Jakes' Doppler spectrum. The normalized Doppler frequency of the channel is denoted as $f_{d} T_{s}$. Simulations were carried out to evaluate the performance of the integer QP relaxation based ICI reduction algorithms in terms of bit error rate (BER) and computational complexity. The BER performance of the conventional one-tap equalizer and DF MMSE [3] are provided for comparison purposes. Perfect channel information was assumed, and combined 1-bit-and-2-bit LBDS was adopted to improve system performance. The algorithms were implemented using the MATLAB SeDuMi toolbox [11].

The BER performance of an OFDM system with $f_{d} T_{s}=0.1$ and the bounded constraint relaxation method is shown in Fig. 2. The performance with a one-tap equalizer and a 25-tap DF MMSE [3] are also given for comparison. It can be observed that the one-tap equalizer provides unsatisfactory performance in time-varying channels, but the bounded constraint relaxation methods considerably mitigate the intercarrier interference. The performance can be further improved by employing the LBDS method. Both the $n$-dimensional and 2-dimensional algorithms offer superior performance to that with the DF MMSE, but with higher computational complexity. Because the solution of (14) is an approximation to that of (11), the $n$-dimensional algorithm outperforms the 2-dimensional algorithm, however, it is more complex. For example, at an $E_{b} / N_{0}$ of $25 \mathrm{~dB}$, the DF MMSE has a BER of $9 \times 10^{-5}$, while the 2-dimensional bounded constraint relaxation algorithm with LBDS has a BER of $5 \times 10^{-5}$ (with a $20 \%$ increase in computational complexity). The $n$-dimensional algorithm has a BER of $2.5 \times 10^{-5}$ with LBDS (with a $40 \%$ increase in computational complexity).

The BER performance of the quadratic convex relaxation algorithms is given in Fig. 3. This shows that the quadratic convex relaxation algorithms exhibit behavior similar to that of the bounded constraint relaxation algorithms, and offer better performance than the one-tap equalizer or DF MMSE. However, the performance is a little worse with the quadratic convex relaxation algorithms. This is because the optimization problem in (12) can be obtained by relaxing (11), so one would expect the bounded constraint relaxation algorithm to offer superior performance with the price of slightly higher computational complexity. For example, with $E_{b} / N_{0}=25 \mathrm{~dB}$, the 2-dimensional quadratic convex relaxation algorithm with LBDS has a BER of $7 \times 10^{-5}$ (with a $18 \%$ increase in computational complexity than that with DF MMSE), while the $n$-dimensional algorithm with LBDS offers a BER of $4.5 \times 10^{-5}$ (with a $35 \%$ increase in computational complexity). 
Simulations were also carried out to determine the impact of normalized doppler spread $f_{d} T_{s}$ on performance. The BER of the 2-dimensional bounded constraint relaxation algorithm for $f_{d} T_{s}=0.05,0.1,0.3$ is plotted in Fig. 4. It can be observed that the performance of the 2-dimensional bounded constraint relaxation algorithm degrades as the Doppler spread increases, while time diversity can be achieved after combining with the LBDS method. For example, an $E_{b} / N_{0}$ of $25 \mathrm{~dB}$ is required to achieve a BER of $10^{-4}$ for $f_{d} T_{s}=0.05$ with LBDS, while with $f_{d} T_{s}=0.1$, an $E_{b} / N_{0}$ of $24 \mathrm{~dB}$ is required to achieve the same BER. The required $E_{b} / N_{0}$ is lowered to $22.5 \mathrm{~dB}$ to obtain the same BER with $f_{d} T_{s}=0.3$. This improvement with increasing $f_{d} T_{s}$ can be attributed to the increased temporal diversity introduced by the larger Doppler spread [6]. Similar diversity gain can be realized by employing the quadratic convex relaxation algorithm as well.

\section{CONCLUSions}

In this paper, the OFDM ICI reduction problem was first formulated as a combinatorial optimization problem with integer constraints. Two relaxation methods were then utilized to convert the discrete ML detection problem into convex QP problems. To further reduce the computational complexity, the QP problems were solved by limiting the search to a 2-dimensional subspace. A LBDS method was employed to improve the system performance with slightly increased computational complexity. Simulations were carried out to examine the performance of the proposed ICI reduction algorithms. The results demonstrated that the integer QP relaxation based algorithms provide excellent performance with reasonable computational complexity, and that temporal diversity can be achieved with increased Doppler spread.

\section{REFERENCES}

[1] R. V. Nee and R. Prasad, OFDM for Wireless Multimedia Communications, Artech House, 2000.

[2] Y. G. Li and L. J. Cimini, Jr., "Bounds on the interchannel interference of OFDM in time-varying impairments," IEEE Trans. Commun., vol. 49, no. 3, pp. 401-404, Mar. 2001.

[3] X. Cai and G. B. Giannakis, "Bounding performance and suppressing intercarrier interference in wireless mobile OFDM," IEEE Trans. Commun., vol. 51, no. 12, pp. 2047-2056, Dec. 2003

[4] W.-S. Hou and B.-S. Chen, "ICI cancellation for OFDM communication systems in time-varying multipath fading channels," IEEE Trans. Wireless Commun., vol. 4, no. 5, pp. 2100-2110, Sept. 2005.

[5] M. Russell and G. L. S. Stuber, "Interchannel interference analysis of OFDM in a mobile environment," Proc. IEEE Vehic. Tech. Conf., pp. 820-824, July 1995.

[6] N. J. Bass and D. P. Taylor, "Matched filter bounds for wireless communication over Rayleigh fading dispersive channel," IEEE Trans. Commun., vol. 49, pp. 1525-1528, Sep. 2001.

[7] H. T. Peng, L. K. Rasmussen, and T. J. Lim, "Constrainted maximumlikelihood detection in CDMA," IEEE Trans. Commun., vol. 49, pp. 142152, Jan. 2002

[8] S. Boyd and L. Vandenberghe, Convex Optimization, Cambridge University Press, 2004.

[9] J. G. Proakis, Digital Communications, 4th Ed., McGraw-Hill, 2001.

[10] W.-S. Lu, "Design of FIR digital filters with discrete coefficients via convex programming," Proc. IEEE ISCAS, pp. 1831-1834, May 2005.

[11] Advanced Optimization Laboratory, McMaster University, "SeDuMi 1.1," http://sedumi.mcmaster.ca/.

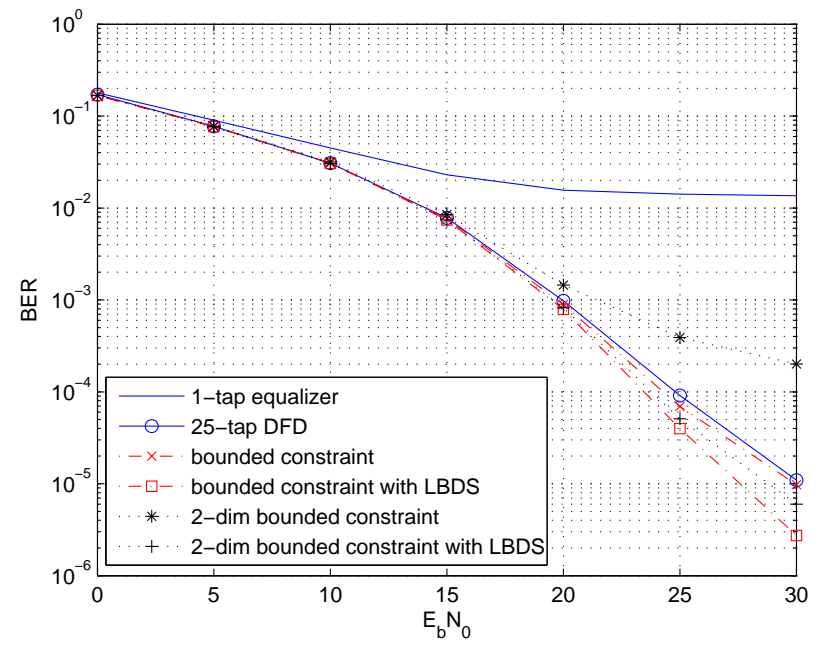

Fig. 2. BER performance of the bounded constraint relaxation method with $f_{d} T_{s}=0.1$.

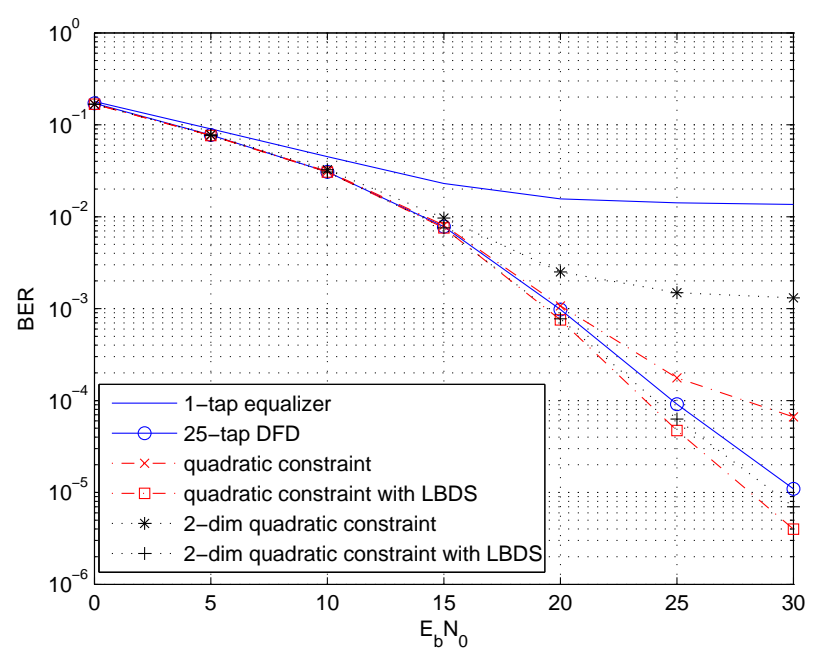

Fig. 3. BER performance of the quadratic constraint relaxation method with $f_{d} T_{s}=0.1$.

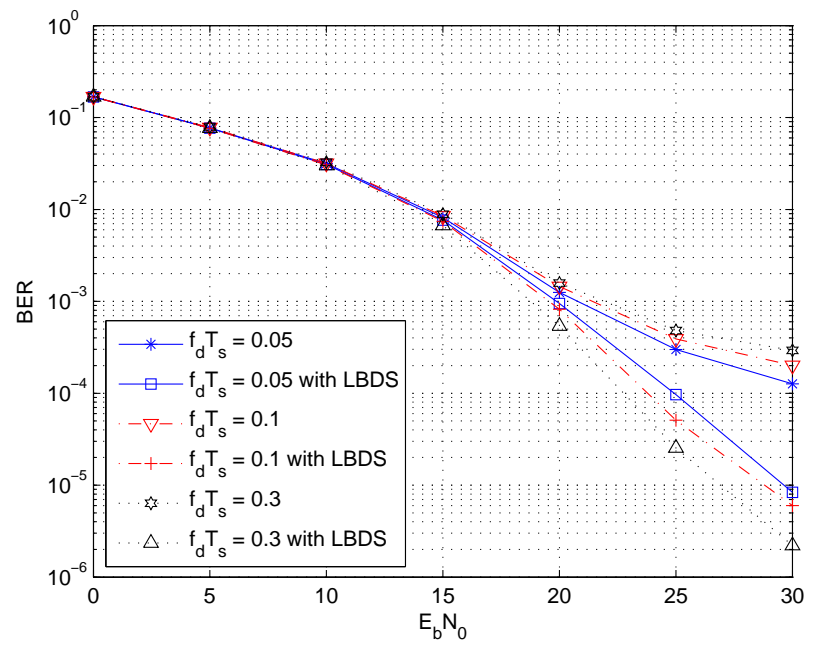

Fig. 4. BER performance of the 2-dimensional bounded constraint relaxation method with different doppler spreads. 